\title{
Correction to: Altered oscillation of Doppler-derived renal and renal interlobar venous flow velocities in hypertensive and diabetic patients
}

\author{
Yusuke Kudo $^{1,2} \cdot$ Taisei Mikami $^{3} \cdot$ Mutsumi Nishida $^{1,2} \cdot$ Kazunori Okada $^{3}$. \\ Sanae Kaga $^{3} \cdot$ Nobuo Masauzi $^{3} \cdot$ Satomi Omotehara $^{1,2} \cdot$ Hitoshi Shibuya $^{1}$. \\ Kaoru Kahata $^{1}$. Chikara Shimizu ${ }^{1}$
}

Published online: 23 October 2017

(C) The Japan Society of Ultrasonics in Medicine 2017

\section{Correction to: J Med Ultrasonics (2017) 44:305-314 DOI 10.1007/s10396-017-0770-0}

In the original publication of this paper the legend of Fig. 1 should read as:

Fig. 1 Pulsed Doppler flow velocity recordings of the aorta (a), right renal artery (b), right renal interlobar artery (c), inferior vena cava (d), right renal vein (e), and right renal interlobar vein (f). $P S V$ peak systolic velocity, $E D V$ end-diastolic velocity, $V_{M A X}$ maximum velocity, $V_{M I N}$ minimum velocity

The second sentence under the heading "Pulsed-wave Doppler sonography" should read as:
Pulsed-wave Doppler sonography was performed under the guide of B-mode or color Doppler imaging with simultaneous electrocardiogram recording (Fig. 1).

In the same paragraph the sentence after the first equation should read as:

We then measured the maximum velocity $\left(V_{\mathrm{MAX}}\right)$ and minimum velocity $\left(V_{\mathrm{MIN}}\right)$ of the inferior vena cava and the right and left renal and renal interlobar veins during inspiration and expiration, respectively, and calculated the mean value for each vein.

The online version of the original article can be found under doi:10.1007/s 10396-017-0770-0.

Taisei Mikami

mikami@hs.hokudai.ac.jp

1 Division of Laboratory and Transfusion Medicine, Hokkaido University Hospital, Kita-14, Nishi-5, Kita-ku, Sapporo 060-8648, Japan

2 Diagnostic Center for Sonography, Hokkaido University Hospital, Kita-14, Nishi-5, Kita-ku, Sapporo 060-8648, Japan

3 Faculty of Health Sciences, Hokkaido University, Kita-12, Nishi-5, Kita-ku, Sapporo 060-0812, Japan 